\title{
Effect of micro- and elevated gravity condition on the evolution of stratification and self-pressurization in a cryogenic propellant tank
}

\author{
S B VISHNU*(B) and BIJU T KUZHIVELI \\ Centre for Advanced Studies in Cryogenics, National Institute of Technology Calicut, Calicut 673601, India \\ e-mail: vishnusb90@gmail.com
}

MS received 13 June 2018; revised 26 September 2018; accepted 11 October 2018; published online 18 February 2019

\begin{abstract}
An efficient way of handling and storing cryogenic propellant is required for future space exploration. In rocketry applications, propellants are stored at subcooled conditions in foam-insulated tanks. Any kind of heat infiltration may lead to stratification and self-pressurization of the tank. The supply of warm propellant beyond the cavitation limit to a turbo-pump is dangerous and hence additional propellant has to be loaded, which affects the payload capacity. The evolution of stratification during lift-off and accelerated conditions and coast phase will be different from those during normal ambient conditions. During lift-off the gravity value can reach up to $6 g$ and microgravity $(\mu \mathrm{g})$ conditions at the coast phase. Hence, accurate prediction of the state of propellant at all stage is required for the successful mission planning. A multiphase axis-symmetric CFD model is developed, which can simultaneously account for heat transfer from the ambient and heat exchanges within the fluids during different gravity conditions. The results show that the self-pressurisation in microgravity condition is due to phase change rather than thermal stratification. The flow velocity will be maximum during lift-off and accelerated condition. Hence, greater self-pressurisation happens during the initial period and reduction in pressure rise rate is noticed later, which is due to turbulence of the fluid.
\end{abstract}

Keywords. Cryogenics; thermal stratification; VOF.

\section{Introduction}

Future space missions require prolonged storage of cryogenic propellants. Nowadays, propellants are stored in foam-insulated tanks. During the mission, there will be heat infiltration into the propellant tank due to solar and space radiation, heat conducted through the support structure, etc. Since propellants are stored at their boiling temperature or subcooled condition, very small heat infiltration itself causes thermal stratification and self-pressurization. When heat is transferred to the propellant tank, the liquid near the sidewall is heated up and a boundary layer will develop. The warm fluid inside the boundary layer will move upwards and is dumped at the liquid-vapour interface. The warm fluid layer developed at the liquid-vapour interface is known as a thermally stratified layer and this phenomenon is known as thermal stratification. Also, a vertical temperature gradient exists along the height of the wall. Since the thermal conductivity of the cryogen is very small, axial heat conduction will be very less and stratification phenomenon will be a stable one. Due to stratification, the state of propellant inside the tank varies and it is very much important to keep the propellant properties in a predefined state for

*For correspondence restarting the cryogenic engine at the conclusion of coast phase.

The self-pressurization in a cryogenic tank is due to thermal stratification. Due to stratification, the temperature at the liquid-vapour interface increases and inside tank pressure will be the saturation value corresponding to stratum temperature. In order to maintain the tank pressure within a limit, successive venting is required. Since the pressurization occurs at a higher rate than normal condition due to stratification, the time between successive venting has to be reduced, which will result in serious problems in cryogen storage and handling. Other reasons are also there to eliminate thermal stratification. For cryogenic rocket engines, the condition of the propellant at the inlet of the propellant feed system or turbo-pump must be within a narrow range. If the inlet temperature is above the cavitation value, cavitation is likely to happen and result in the probable destruction of the flight vehicle.

For the past 60 years, researchers had studied thermal stratification, which includes experimental investigation, mathematical models and numerical simulation. Tatom et al [1] conducted experiments on a liquid hydrogen storage tank to study the evolution of stratification. Fan et al [2] studied the effect of sidewall heating and bottom wall heating on stratification and found that if bottom heating is applied properly on a propellant tank, 
considerable reduction on stratification is possible. Ludwig et al [3] conducted experiments on a partially filled liquid nitrogen tank subjected to periodic lateral forces and found that initial pressure drop is larger in the case of sloshing condition and suddenly comes to a stop. The sudden stop in the pressure drop was due to the formation of a mixed layer below the liquid surface. A mathematical model was developed to predict the stratification at rotating and reduced gravity environments by Justin et al [4]. A modified correlation was also developed for stratum growth and stratum temperature determination under rotating and reduced gravity environments. The effect of insulation thickness, subcooling and wind velocity on the evolution of stratification was investigated by Joseph et al [5], who reported that higher tank pressure leads to higher liquid stratified mass. Fu et al [6] developed a numerical model to predict stratification, which focused on evaporation and its effect on vapour pressure under microgravity conditions. Extensive studies are required to predict the stratification in the micro- and higher gravity condition.

\section{Physical model and governing equations}

To understand the fluidic and thermodynamic phenomena during the self-pressurization period, a multiphase $2 \mathrm{D}$ axissymmetric cylindrical tank partially filled with liquid hydrogen is considered as shown in figure 1. Liquid and vapour hydrogen were present at their saturation condition

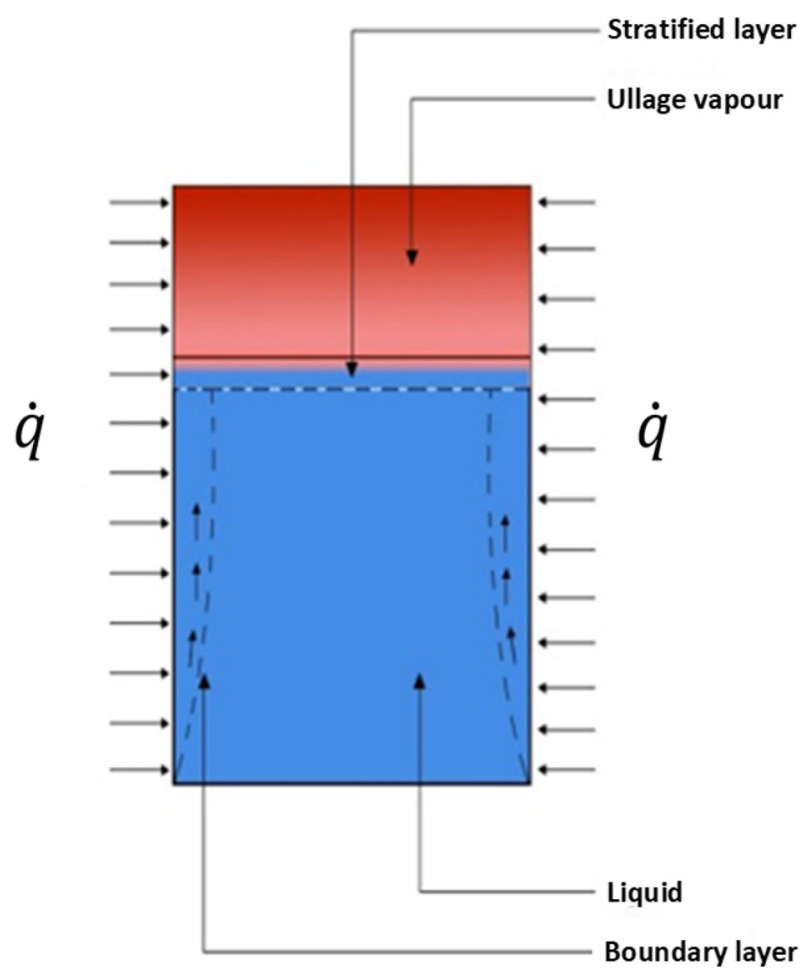

Figure 1. Schematic of thermal stratification phenomenon. corresponding to atmospheric pressure $(101.3 \mathrm{kPa})$. A timedependent VOF (Volume Of Fluid) method is used for the simulation. Modelling of two or more immiscible fluids by solving a single set of momentum equation is possible by this method. Tracking the shape and interface between liquid and vapour is accomplished by the solution of the continuity equation for the volume fraction of the two phases. The sum of the volume fraction of two phases in each control volume becomes unity. Since the buoyancy effect is predominant, the density variation due to temperature change is obtained by Boussinesq approximation.

Governing equations are as follows:

conservation of mass

$$
\frac{\partial \rho}{\partial t}+\nabla \cdot(\rho \vec{V})=0
$$

conservation of momentum

$$
\begin{aligned}
\frac{\partial(\rho \vec{V})}{\partial t}+\nabla \cdot(\rho \vec{V} \vec{V})= & -\nabla P-\rho \beta \vec{g}\left(T-T_{0}\right) \\
& +\nabla \cdot\left[\mu_{e f f}\left(\nabla \vec{V}+\nabla \vec{V}^{T}\right)\right]+\vec{F}
\end{aligned}
$$

conservation of energy

$$
\frac{\partial(\rho C T)}{\partial t}+\nabla \cdot(\vec{V}(\rho C T+P))=\nabla \cdot\left(K_{e f f} \nabla T\right)+S_{h}
$$

where $C$ and $T$ represent specific heat and temperature, respectively. $\vec{F}$ is the resultant surface tension force at the interface and $s_{h}$ is the energy source of heat exchange related to phase change process. Here the surface tension is modelled via Continuum Surface Force model [7]. In this model, the surface force is transformed into volume force $\left(F_{v o l}\right)$

$$
F_{v o l}=\sigma_{l v} \frac{\alpha_{l} \rho_{l} K_{v} \nabla \alpha_{v}+\alpha_{v} \rho_{v} K_{l} \nabla \alpha_{l}}{0.5\left(\rho_{l}+\rho_{v}\right)}
$$

where $\sigma_{l v}$ is the interfacial surface tension coefficient. $K_{v}$ and $K_{l}$ are, respectively, the surface curvature of vapour and liquid, which are defined as follows:

$$
K_{l}=\frac{\nabla \alpha_{l}}{\left|\nabla \alpha_{l}\right|}, \quad K_{v}=\frac{\nabla \alpha_{v}}{\left|\nabla \alpha_{v}\right|}
$$

where $\alpha_{l}$ and $\alpha_{v}$ represent the volume fraction of liquid and vapour. This method has been widely used by many investigators [8-10].

To determine the variation of interface, VOF method is adopted, in which the changes happening at the interface of each cell are located by solving the continuity equation for the volume fraction of second phase:

$$
\frac{\partial}{\partial t}\left(\alpha_{v} \rho_{v}\right)+\nabla \cdot\left(\alpha_{v} \rho_{v} \vec{V}\right)=\dot{m} .
$$


In this equation, $\dot{m}$ represents the phase change due to evaporation or condensation at the interface. It is considered as a source term in the continuity equation. The Lee phase change model [11] is applied to consider this mass transfer and is described by

$$
\begin{array}{cl}
\dot{m}=\frac{r_{l} \rho_{l} \alpha_{l}\left(T_{l}-T_{\text {sat }}\right)}{T_{\text {sat }}} & T_{l} \geq T_{\text {sat }} \\
\dot{m}=\frac{r_{v} \rho_{v} \alpha_{v}\left(T_{v}-T_{\text {sat }}\right)}{T_{\text {sat }}} & T_{v}<T_{\text {sat }} .
\end{array}
$$

According to the tank pressure, the saturation temperature $T_{\text {sat }}$ will change. The coefficient ' $r$ ' is the mass transfer time parameter with the unit $s^{-1}$. This coefficient must be finely tuned to match experimental data. According to Lee [11] and Sandra et al [12] the value of $r$ can be selected as 0.1 .

The energy source term $S_{h}$ can be defined as follows:

$$
S_{h}=\dot{m} L_{h}
$$

where $L_{h}$ is the latent heat of liquid hydrogen.

Since temperature change is negligibly small, all the properties except density remain constant and property values are determined by the number of individual phases in each control volume.

The important properties are

thermal conductivity

$$
k=\alpha_{l} k_{l}+\left(1-\alpha_{l}\right) k_{v}
$$

density

$$
\rho=\alpha_{l} \rho_{l}+\left(1-\alpha_{l}\right) \rho_{v}
$$

absolute viscosity

$$
\mu=\alpha_{l} \mu_{l}+\left(1-\alpha_{l}\right) \mu_{v}
$$

and specific heat

$$
C_{p}=\frac{1}{\rho}\left[\alpha_{l} \rho_{l} C_{p l}+\left(1-\alpha_{l}\right) \rho_{\nu} C_{p v}\right] .
$$

The assumptions used are as follows.

(1) The liquid-vapour interface temperature represents the saturation value corresponding to the pressure of the cryogenic tank.

(2) The heat transfer from ambient is considered as steady.

(3) The heat transfer across the liquid-vapour interface is due to natural convection and steady.

(4) The tank pressure is assumed to be uniform throughout the ullage.

The applied boundary conditions are as follows.

On the sidewalls

$$
-k \frac{\partial T}{\partial n}=q_{w} .
$$

At the bottom and top surfaces, the walls are adiabatic:

$$
\frac{\partial T}{\partial n}=0
$$

where $n$ is the direction normal to the wall.

\section{Numerical implementation}

A two-dimensional cylindrical tank with $0.5 \mathrm{~m}$ diameter and $1 \mathrm{~m}$ height is used for the simulation. The tank wall thickness is $0.003 \mathrm{~m}$. Commercial CFD package Ansys 15 is used for solving the conservation equations. Because of the nature of geometry, boundary conditions and physics of the phenomenon, axis-symmetric condition is selected. The nature of the flow can be identified by defining a nondimensional number called Grashoff number. Under all operating conditions, the flow is always turbulent in nature. Hence, $k$ - $\varepsilon$ turbulence model with enhanced wall function approach is applied. A constant heat flux of $10 \mathrm{~W} / \mathrm{m}^{2}$ is applied on the left sidewall. The pressure-velocity coupling algorithm selected is SIMPLEC (Semi-Implicit Method for Pressure-Linked Equation-Consistent). The converged solution is easy to achieve by this method than by a SIMPLE algorithm. The body-force-weighted average scheme is used for solving the momentum equation. For tracking the liquid-vapour interface, the Geometric Reconstruction Scheme is applied. Since the problem is transient in nature, a time step of $0.001 \mathrm{~s}$ is selected in such a way that Courant number is less than 0.1. The liquidvapour interface movement is predicted by the VOF method. In each cell, volume fraction is defined in such a way that the sum of both liquid and vapour volume fractions becomes unity.

Three grid systems with mesh numbers 15288, 22893 and 30671 are used to make the calculation results tend to the real situation. Table 1 shows the variation of pressure inside the tank with mesh number. The self-pressurizations

Table 1. Effect of mesh size on self-pressurisation.

\begin{tabular}{cccc}
\hline & \multicolumn{3}{c}{ Tank pressure (Pa) } \\
\cline { 2 - 4 } Time (s) & Grid no. 15288 & Grid no. 22893 & Grid no. 30671 \\
\hline 10 & 101642 & 101690 & 101720 \\
20 & 102057 & 102027 & 102030 \\
30 & 102355 & 102392 & 102442 \\
40 & 102610 & 102765 & 102838 \\
50 & 102986 & 103134 & 103240 \\
60 & 103214 & 103426 & 103555 \\
70 & 103456 & 103640 & 103803 \\
80 & 103614 & 103801 & 103929 \\
90 & 103875 & 104060 & 104171 \\
100 & 104012 & 104350 & 104483 \\
\hline
\end{tabular}


for the two finest grids are almost the same as the maximum pressure difference is less than $165 \mathrm{kPa}$. For the mesh number of 30671 , the solution is too time-consuming. Hence, the grids with 22893 quadrilateral grid elements are used; successively increasing mesh numbers towards the wall are selected for the present simulation. The maximum value of $y+$ near the solid wall obtained is 30 , which is within the permitted range of $k-\varepsilon$ turbulence model with enhanced wall function.

\section{Results and discussion}

\subsection{Validation}

Validation of the numerical model is required to consider the self-pressurization of the cryogenic tank. The results obtained from the numerical model are validated with the experimental results obtained by Seo and Jeong [13]. The experimental set-up consists of a cylindrical tank with a diameter of $0.201 \mathrm{~m}$ and height $0.213 \mathrm{~m}$. The cryogen used was liquid nitrogen with $50 \%$ fill level. A constant heat flux of $1.2 \mathrm{~W}$ was applied on the left sidewall. The liquid and vapour were at an initial pressure of $99 \mathrm{kPa}$ and temperature corresponding to the saturation value. The self-pressurization for a time period of $100 \mathrm{~s}$ was captured and compared to numerical results. In the initial period, the pressure obtained from simulation is found to be lesser than the experimental value and becomes almost the same for the remaining periods. Hence, it can be concluded that the numerical predictions are in good agreement with the experimental results (figure 2).

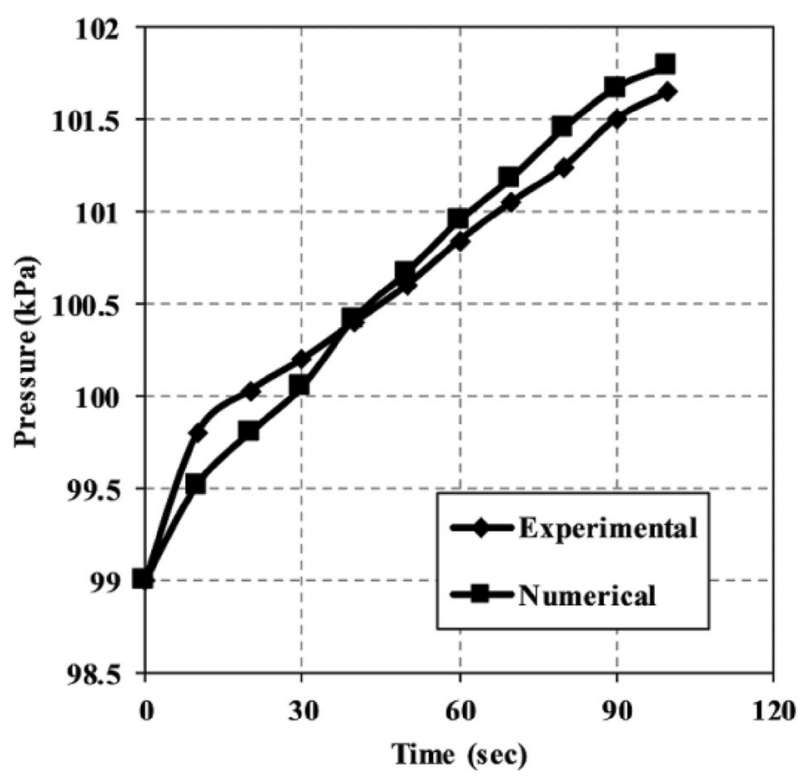

Figure 2. Validation of the numerical model.

\subsection{Evolution of stratification}

A numerical model had been developed to study the evolution of stratification in a liquid hydrogen tank of $0.5 \mathrm{~m}$ diameter and $1 \mathrm{~m}$ height. The tank is partially filled with liquid hydrogen at saturation condition corresponding to ambient pressure. Since the situation demands axis-symmetric condition, the heat flux applied is restricted to left sidewall only.

As the heat flux is applied, the liquid near the left wall absorbs the heat and begins to expand. A boundary layer is developed near the left sidewall; warm liquid in the boundary layer moves upwards and forms a warm layer at the liquid-vapour interface. This warm layer is known as the thermally stratified layer. During the evolution of stratification, the rate of evaporation is very small. Once the thermally stratified layer is formed, it increases the rate of evaporation; more amount of liquid evaporates and pressure starts building.

Initial pressure is fixed at $101.3 \mathrm{kPa}$ and pressure variation for a time period of $150 \mathrm{~s}$ is captured. Since the boundary layer fluid is continuously heated up and dumped into the liquid-vapour interface, pressure increases. A warm layer of fluid at the liquid-vapour interface is visible in figure 3. Since heat flux is applied on the left wall, a warmer layer of fluid in the boundary layer, as well as stratum layer, is formed and the maximum liquid temperature obtained is $20.390 \mathrm{~K}$ at the end of $150 \mathrm{~s}$. Compared with the stratum layer, the bulk liquid temperature is always less and uniform. The rise in pressure inside the tank seems to be uniform in nature as shown in figure 4 . During a time span of $150 \mathrm{~s}$, the pressure rise obtained was around $3.95 \mathrm{kPa}$.

\subsection{Self-pressurization in microgravity condition}

The evolution of stratification in microgravity condition was studied for a partially filled liquid hydrogen tank. A flat liquid-vapour interface is initialized and reduced gravity value is applied to the simulation. The fluid is assumed to be in equilibrium and considered as a Newtonian fluid. Apart from natural convection phenomenon, self-pressurization phenomenon in microgravity condition is due to phase change. As sidewall heat flux is applied, instead of upward buoyancy force, local hot spots are created and the liquid becomes superheated. The superheated liquid starts evaporating and local vapour zones are created. As time progresses, the vapour region grows and detaches from the tank wall (figure 5). These vapour zones move to the interface under the coupling of surface tension and buoyancy force. Some vapour zones were found to merge together before reaching the interface.

In microgravity conditions, it is difficult to transfer heat to the liquid-vapour interface and form a stratified layer. The applied heat will accumulate near the tank wall and will heat the liquid locally. When compared with the 


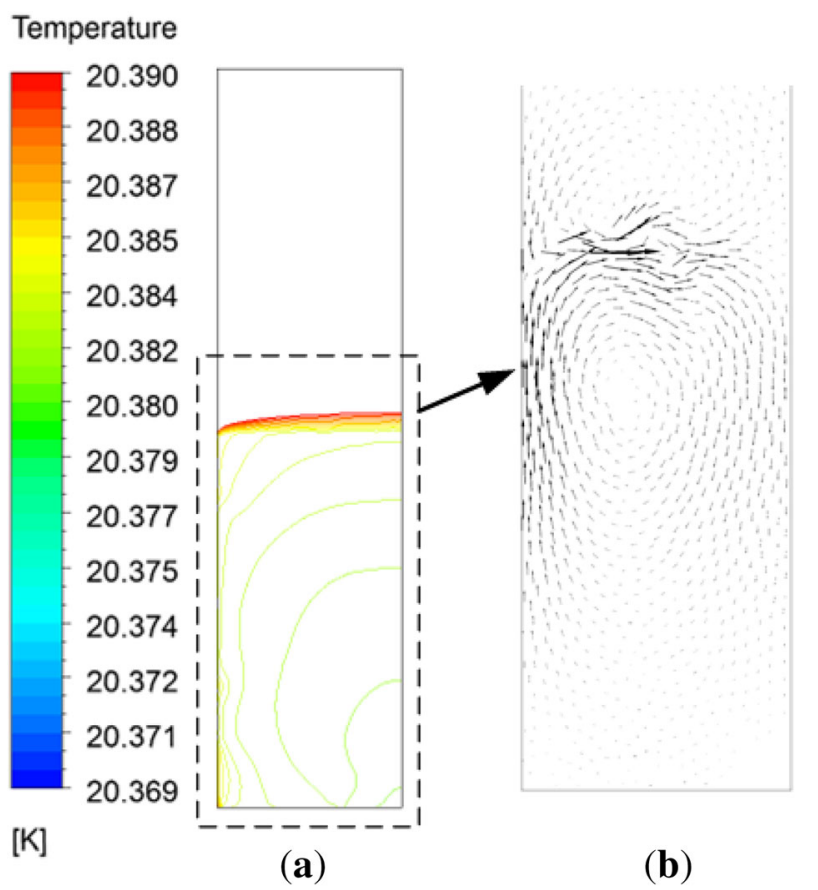

Figure 3. (a) Evolution of stratification and (b) velocity vector.

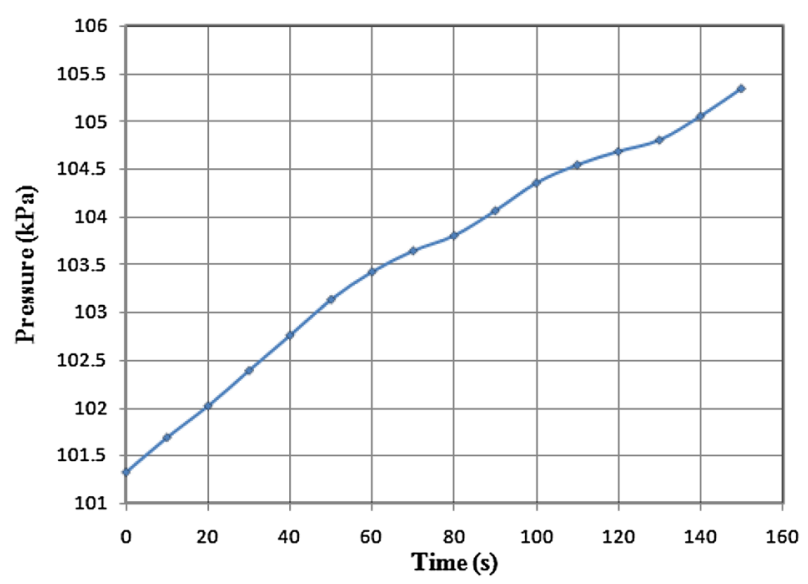

Figure 4. Self-pressurization in the propellant tank.

natural convection, the heat transfer ability in this mentioned condition will be less. When the temperature of the heated liquid increases and exceeds the saturation temperature corresponding to the tank pressure, evaporation takes place and it becomes the major heat transfer mode. Then heat transfer coefficient increases and more amount of heat is transferred into the liquid.

\subsection{Influence of vehicle acceleration}

During the mission, the vehicle experiences extreme gravity conditions: micro- or zero gravity conditions at the coast phase and higher gravity conditions during lift-off and acceleration phase. In these conditions, the nature of heat transport through the liquid will be different. Figure 6 shows the nature of the stratification phenomenon at different gravity conditions after $165 \mathrm{~s}$. Under microgravity conditions $\left(10^{-3} g_{\mathrm{o}}\right)$, a lot of vapour zones in the liquid regions are visible. Because of the microgravity conditions, the velocity is found to be too low (figure 8) and local heating of the fluid takes place near the tank wall. When the enthalpy value exceeds the required energy for phase change, local boiling takes place and small bubble formation takes place. They move upwards due to the buoyancy effect and surface tension, transfer heat to the vapour region and pressure inside the tank increases. Hence, a maximum temperature of $20.410 \mathrm{~K}$ is obtained (figure 7) for stratification in microgravity condition. From figure 8, comparatively undisturbed bulk liquid zones are visible. The fluid motion is visible only near the tank wall and interface.

Under normal gravity conditions $\left(g_{\mathrm{o}}\right)$, the evolution of stratification is due to the natural convection. Due to sidewall heating, a boundary layer flow develops near the tank wall and warm fluid moves upwards, forming a layer known as a stratified layer. Under normal gravity conditions, there will be convection current, which assists the recirculation of fluid through the bulk liquid and bulk liquid temperature increases. Hence, we can see small bubble formation all over the liquid and the stratum temperature is $20.390 \mathrm{~K}$. A maximum velocity of around $0.146 \mathrm{~m} / \mathrm{s}$ is
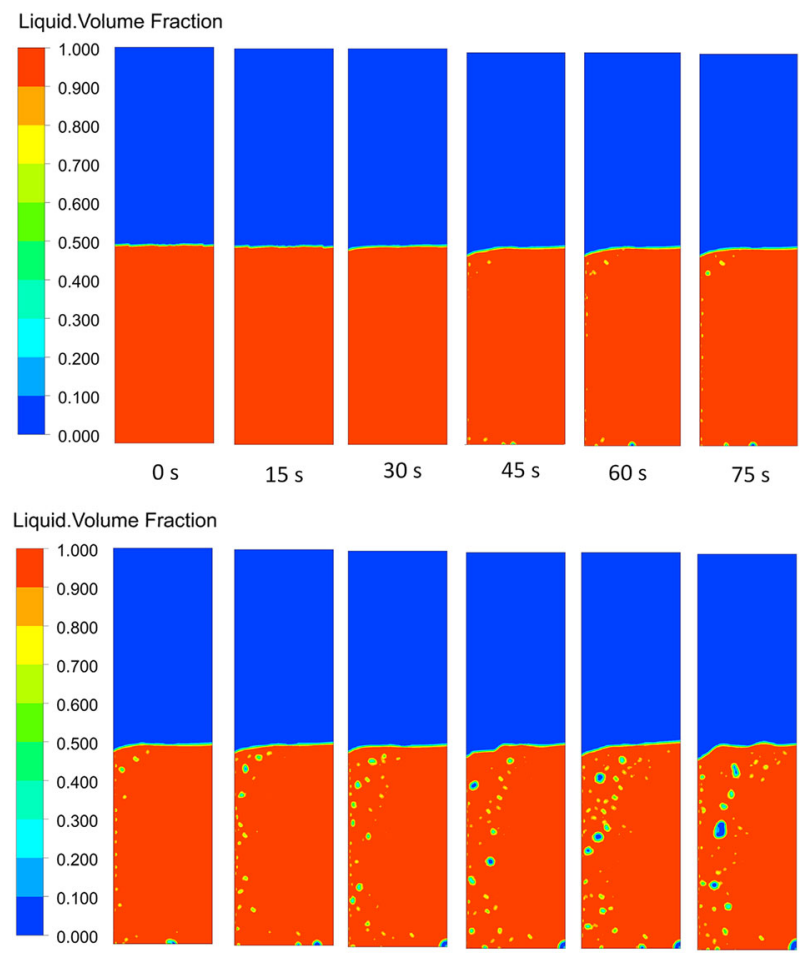

$90 \mathrm{~s}$

$120 \mathrm{~s}$

$135 \mathrm{~s}$

$150 \mathrm{~s}$

$165 s$

Figure 5. Liquid volume fraction in microgravity condition. 


\section{Liquid.Volume Fraction}

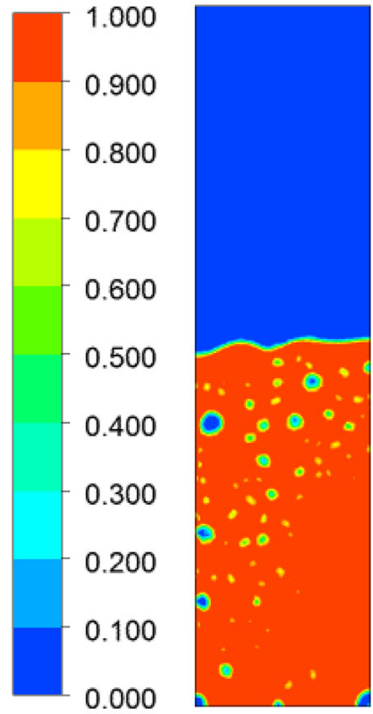

(a) $10^{-3} \mathrm{~g}_{0}$

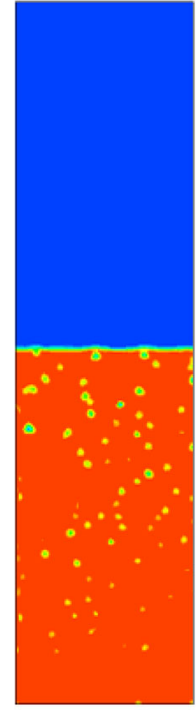

(b) $\mathrm{g}_{0}$

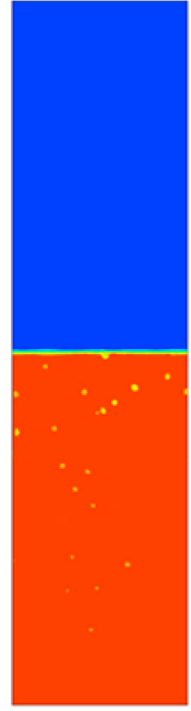

(c) $6 g_{0}$
Figure 6. Liquid volume fraction after $165 \mathrm{~s}$ in different gravity conditions.

obtained near the tank wall as well as the bottom of the tank. It implies that there is a greater influence on the flow of fluid through the buoyancy effect as well as recirculation convection currents.

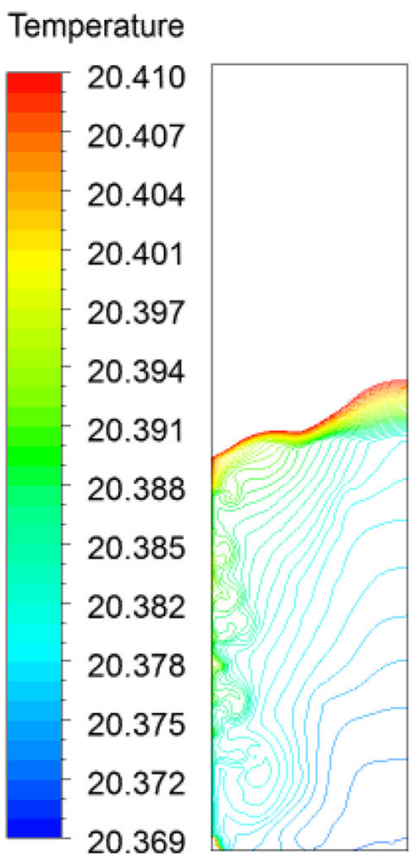

$[\mathrm{K}]$

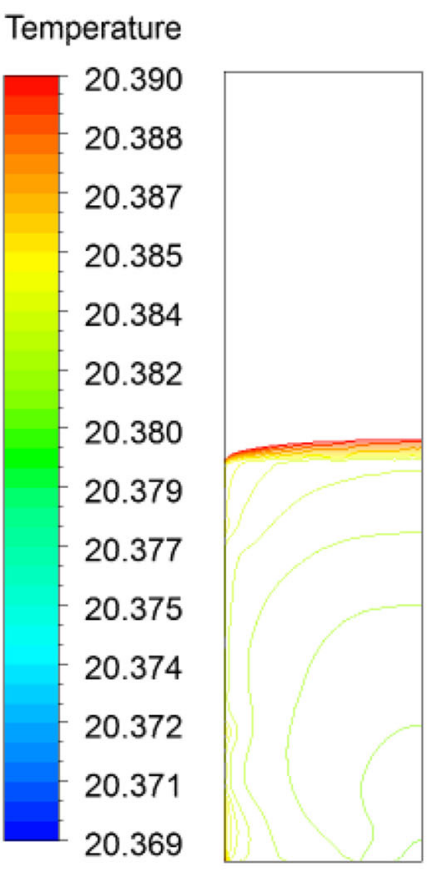

$[\mathrm{K}]$ (a) $10^{-3} \mathrm{~g}_{0}$
The evolution of stratification during higher gravity condition is entirely different from that during microgravity condition. Since the gravity value is higher, the flow will be highly turbulent in nature. So chances of local heating of fluid are very less because of the vigorous turbulence created during the acceleration of the vehicle. In these type flow situations, the bubble formation is very less and chances of phase change due to stratification will be very less. Compared with micro- and normal gravity conditions, the stratum temperature developed in the tank is $20.382 \mathrm{~K}$, which is the least. Since the heat flux remains the same, more amount of heat will be transferred to the bulk liquid. A maximum velocity of $0.333 \mathrm{~m} / \mathrm{s}$ is obtained which is the highest among the microgravity and normal gravity conditions.

\subsection{Evolution of pressure}

The self-pressurization of the tank is found out at three gravity conditions (figure 9). The pressure builds up almost constantly at microgravity conditions. This is because of the phase change happening inside the tank. Since constant heat flux is applied throughout, more amount of liquid boils and pressure increases. In higher gravity condition $(6 g)$, the initial pressure rise rate was somewhat higher than microand normal gravity conditions. It is due to the turbulent nature of flow and higher fluid velocity, which will assist the stratification phenomenon. However, after stratification,

igure 7. Liquid temperature distribution in different gravity conditions. 
Velocity

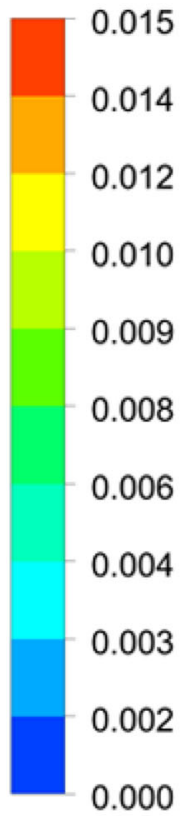

$\left[\mathrm{m} \mathrm{s}^{\wedge}-1\right]$
Velocity

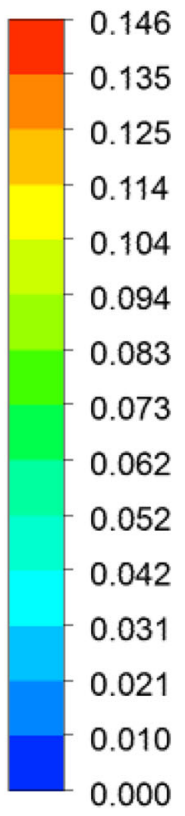

$\left[\mathrm{m} \mathrm{s}^{\wedge}-1\right]$

(a) $10^{-3} \mathrm{~g}_{0}$

\section{Velocity}

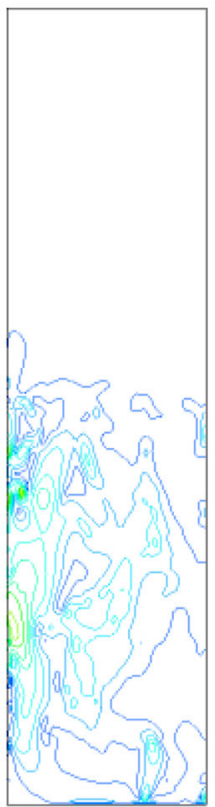

(b) $g_{0}$

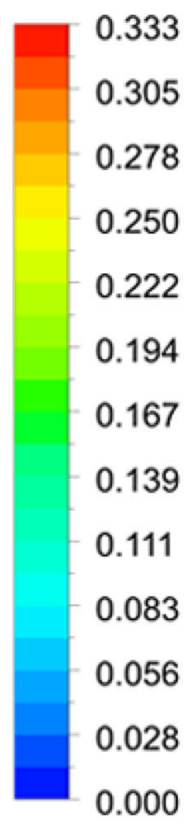

$\left[\mathrm{m} \mathrm{s}^{\wedge}-1\right]$

Figure 8. Velocity distribution after $165 \mathrm{~s}$ in different gravity conditions.

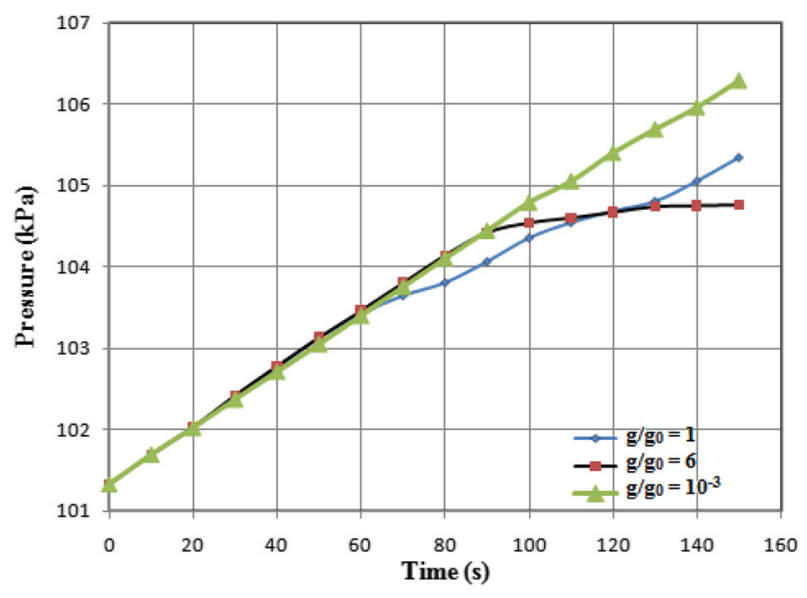

Figure 9. Self-pressurization in a partially filled liquid hydrogen tank for different gravity conditions.

the value of pressure seems to decrease and almost maintains a constant value. It is due to the fluid turbulence and good mixing of the bulk fluid with the stratum layer. However, the bulk temperature will be always high in these conditions. In normal gravity condition, the self-pressurization is due to stratification only. Once the stratum layer formation takes place, the pressure continuously increases but the pressure value is lower than that in microgravity condition.

\section{Conclusions}

A numerical model is developed to understand the thermodynamic and fluidic phenomena happening inside a propellant tank that is partially filled with liquid hydrogen. VOF method is used and heat and mass transfer models are also incorporated to study the effect of phase change on thermal stratification. The model is validated with the experimental results available in the literature. Under microgravity conditions, it is found that the self-pressurization is due to phase change rather than natural convection. Small vapour zones emerge and couple together to transfer energy from liquid to vapour. Hence, the pressure rise is found to be larger in microgravity condition compared with normal and higher conditions. In higher gravity conditions, after a sudden rise in pressure at the initial condition, the pressure level drops. It is due to the turbulence and mixing of the stratum liquid with the bulk liquid. Since the geometry used is a perfect cylinder, further studies about stratification are required by considering actual propellant tank geometries.

\section{Nomenclature}
$C \quad$ specific heat, $\mathrm{J} / \mathrm{kg} \mathrm{K}$
$C p \quad$ specific heat at constant pressure, $\mathrm{J} / \mathrm{kg} \mathrm{K}$
$F$ body force, $\mathrm{N} / \mathrm{m}^{3}$ 
K

$L_{h}$

$\dot{m}$

$n$

$P$

$\dot{q}$

$r \quad$ mass transfer time parameter, $\mathrm{s}^{-1}$

$S_{h} \quad$ energy source term

$T$ temperature, $\mathrm{K}$

$t$ time, s

$\vec{V} \quad$ velocity vector, $\mathrm{m} / \mathrm{s}$

\section{Greek symbols}

$\alpha$ volume fraction

$\beta$ thermal expansion coefficient, $\mathrm{K}^{-1}$

$\mu$ absolute viscosity, $\mathrm{Pa} \mathrm{s}$

$\rho$ density, $\mathrm{kg} / \mathrm{m}^{3}$

$\sigma$ surface tension, $\mathrm{N} / \mathrm{m}$

\section{Subscripts}

h heat

1 liquid

sat saturation

$\mathrm{v}$ vapour

vol volume

\section{References}

[1] Tatom J W, Brown W H, Knight L H and Coxe E F 1964 Analysis of thermal stratification of liquid hydrogen in rocket propellant tanks. Adv. Cryog. Eng. 9: 265-272
[2] Fan S C, Chu J C and Scott L E 1969 Thermal stratification in closed cryogenic containers. Adv. Cryog. Eng. 14: 249-257

[3] Ludwig C, Dreyer M E and Hopfinger E J 2013 Pressure variations in a cryogenic liquid storage tank subjected to periodic excitations. Int. J. Heat Mass Transf. 66: 223-234

[4] Justin O, Daniel K and Paul S 2007 Effect of isogrid roughness on thermal stratification. In: Proceedings of the 43rd AIAA/ASME/SAE/ASEE Joint Propulsion Conference and Exhibit, American Institute of Aeronautics and Astronautics

[5] Joseph J, Agrawal G, Agarwal D K, Pisharady J C and Sunil Kumar S 2017 Effect of insulation thickness on pressure evolution and thermal stratification in a cryogenic tank. Appl. Therm. Eng. 111: 1629-1639

[6] Fu J, Sunden B, Chen X and Huang Y 2015 Influence of phase change on self-pressurization in cryogenic tanks under microgravity. Appl. Therm. Eng. 87: 225-233

[7] Brackbill J U, Kothe D B and Zemach C 1992 A continuum method for modeling surface tension. J. Comput. Phys. 100: 335-354

[8] Sun D, Xu J and Chen Q 2014 Modeling of the evaporation and condensation phase change problems with FLUENT. Numer. Heat. Transf. Part B 66: 326-342

[9] Sun D, Xu J and Wang L 2012 Development of a vaporliquid phase change model for volume-of-fluid method in FLUENT. Int. Commun. Heat Mass Transf. 39: $1101-1108$

[10] Pan Z, Weibel J A and Garimella S V 2015 Spurious current suppression in VOF-CSF simulation of slug flow through small channels. Numer. Heat. Transf. Part A 67: 1-12

[11] Lee H 1980 A pressure iteration scheme for two-phase flow modeling. In: Veziroglu $\mathrm{T} \mathrm{N}$ (Ed.) Multiphase transport: fundamentals, reactor safety, applications, vol. 1, pp. 407-432

[12] Sandra C K, De Schepper S C K, Heynderickx G J and Marin G B 2009 Modeling the evaporation of a hydrocarbon feedstock in the convection section of a steam cracker. Comput. Chem. Eng. 33: 122-132

[13] Seo M and Jeong S 2010 Analysis of self-pressurization phenomenon of cryogenic fluid storage tank with thermal diffusion model. Cryogenics 50: 549-555 